\title{
Diagnostic Role of Cardiovascular Magnetic Resonance Imaging in Dilated Cardiomyopathy
}

\author{
Nehal Singla ${ }^{1}$ Shibani Mehra ${ }^{1} \quad$ Umesh C. Garga ${ }^{1}$ \\ ${ }^{1}$ Department of Radio-Diagnosis, PGIMER and RML Hospital, New \\ Delhi, India
}

Indian J Radiol Imaging 2021;31:116-123.

\begin{abstract}
Address for correspondence Nehal Singla, MBBS, MD, Department of Radio-Diagnosis, PGIMER and RML Hospital, New Delhi 11001, India (e-mail: nehalsingla19@gmail.com).
\end{abstract}

\begin{abstract}
Keywords

- cardiac magnetic resonance imaging

- dilated cardiomyopathy

- late gadolinium enhancement

Aims The purpose of the study was to compare the accuracy of cardiac magnetic resonance (CMR) with echocardiography for the evaluation of ventricular dysfunction in patients of dilated cardiomyopathy (DCM). Further, we evaluated the potential of CMR for myocardial tissue characterization.

Design Prospective observational.

Materials and Methods A total of 30 patients with suspected DCM prospectively underwent cardiac magnetic resonance (MR) using a 1.5 Tesla MR scanner, with appropriate phased-array body coils. Dynamic sequences after injection of $0.1 \mathrm{mmol} / \mathrm{kg}$ of body weight of gadolinium-based intravenous contrast (Magnevist) were acquired for each patient, after which delayed images were obtained at an interval of 12 to 15 minutes. Myocardial tagging was performed in all patients for assessment of wall motion abnormalities. Each MR examination was interpreted with two radiologists for chamber dimensions and ventricular dysfunction as well as morphologic characteristics with disagreement resolved by consensus. All patients included in the study were taken up for MR evaluation after cardiological evaluation through echocardiography and the results for both the studies were compared. Data were analyzed through standard statistical methods.

Conclusion CMR is a comprehensive diagnostic tool, which can estimate the ventricular function more precisely than echocardiography. CMR reliably differentiates between ischemic and nonischemic etiologies of DCM based on patterns of late gadolinium enhancement (LGE) and based on the presence or absence of LGE, which helps to estimate the degree of myocardial fibrosis. Thereby it can be a useful tool in establishing risk stratification, predicting prognosis, and thus instituting appropriate therapy in DCM patients.
\end{abstract}

\section{Introduction}

Dilated cardiomyopathy (DCM) is amongst the most common causes of heart failure. Cardiomyopathy is a heterogeneous group of diseases of myocardium associated with mechanical or electrical dysfunction that usually (but not

published online May 31, 2021
DOI https://doi.org/

$10.1055 / \mathrm{s}-0041-1730133$

ISSN 0971-3026 invariably) exhibit appropriate ventricular hypertrophy or dilatation. ${ }^{1}$ The estimated prevalence of DCM is $1: 2,500 .{ }^{2}$ DCM is broadly classified into ischemic DCM and nonischemic DCM (NIDCM) groups with the NIDCM being further classified based on the etiology into primary and secondary NIDCM. Primary NIDCM includes genetic etiology (familial), 
infectious etiology (mainly viral infections caused by coxsackievirus, adenovirus, parvovirus, and human immunodeficiency virus), and sporadic cases. Secondary causes of NIDCM include autoimmune and systemic diseases (sarcoidosis and amyloidosis), mitochondrial disorders, chronic alcoholism, and drugs (anthracyclines and ingestion of heavy metals) ${ }^{3-6}$ Ischemic DCM results from coronary artery disease leading to myocardial infarction (MI) and subsequent myocardial damage.

Cardiac magnetic resonance (CMR) imaging is a modality that detects morphological changes associated with DCM and simultaneously assesses the myocardial function as well as myocardial perfusion. Contrast-enhanced CMR (CECMR) enables the detection and characterization of myocardial damage. ${ }^{7}$ The chief advantage of CMR imaging is its excellent spatial resolution and multiplanar imaging capability which can provide details of cardiac chamber anatomy with simultaneous information about the status of myocardial perfusion and about the impairment of diastolic and systolic functions.

Delayed gadolinium-enhanced magnetic resonance imaging (MRI) can differentiate myocardial ischemia from myocardial fibrosis. In fact, contrast-enhanced MR is currently the mainstay for differentiation of ischemic and nonischemic subtypes of DCM based on the patterns of late gadolinium enhancement (LGE) of the myocardium. ${ }^{8}$ This differentiation is extremely important in patients of DCM to separate the NIDCM group from Ischemic DCM for instituting appropriate clinical management. Since myocardial perfusion and cardiac function can be reliably assessed with CMR, it has become the preferred modality for evaluating the prognosis in patients of DCM, thereby further assisting in therapeutic triage. ${ }^{9}$

\section{Methods}

A prospective observational study was conducted from the period of November 2014 to March 2016 in the Department of Radio-Diagnosis in collaboration with the Department of Cardiology and the Department of Medicine of Ram Manohar Lohia Hospital and PGIMER, Delhi, respectively. A total of 30 patients of suspected DCM were evaluated by CMR. Patients presenting with dyspnea (Grade II/III NYHA [New York Heart Association]) and/or those with ECG changes of infranodal conduction abnormalities were included in the study group. All patients were also assessed by transthoracic echocardiography (ECG) for global or regional impairment of left ventricular (LV) systolic function, as well as for chamber enlargement as per American Society of Echocardiography guidelines.

CMR was performed in all patients using a 1.5 Tesla MR scanner, with appropriate phased-array body coils. The standard cardiac protocol included gradient imaging in short axis, two-chamber, and four-chamber views along with T2 sequences and STIR (short tau inversion recovery) sequences for myocardial tissue characterization. Dynamic sequences after injection of $0.1 \mathrm{mmol} / \mathrm{kg}$ of body weight of gadolinium-based intravenous contrast were acquired for each patient.

Delayed images were obtained at an interval of 12 to 15 minutes after the dynamic sequences. The inversion time was adjusted iteratively with different values to null the normal myocardium. Myocardial tagging was performed in all patients for assessment of wall motion abnormalities. Each MR examination was interpreted by two radiologists with disagreement resolved by consensus. Data were analyzed using standard statistical methods.

All patients included in the study were followed up at intervals of 6 weeks, 3 months, and 6 months by the cardiologist for any symptomatic deterioration with repeat chest X-ray, ECG as well as Holter monitoring performed at both 3 and 6 months for those patients who developed arrhythmias during the follow-up period. A record of the treatment undergone (conservative or intervention) and the number of hospitalizations (due to decompensated heart failure or other causes) for each patient was maintained. Fatal outcomes if any were documented.

\section{Results}

The study consisted of 30 subjects with a mean age of presentation 38.9 years. A total of $26 \%$ of the patients were in the 5 th decade of their life and $23.3 \%$ patients were in the 4 th decade. Out of the 30 patients included in the study, 20 were males (66.6\%) and 10 were females (33.33\%). Male to female ratio was $2: 1$.

A total of $46.6 \%$ ( $n=14$ ) of patients belonged to clinical NYHA class II while $30 \%(n=9)$ had NYHA class III symptomatology at the time of CMR. On ECG, nonspecific ST segment and $\mathrm{T}$ wave abnormalities were present in 11 patients (36\%), 5 patients (16.66\%) had supraventricular tachycardia, 7 patients (23.33\%) showed sinus tachycardia, and 7 patients (23.33\%) showed intraventricular conduction delays in the form of $\mathrm{QRS}$ widening.

The functional parameters evaluated by CMR included ejection fraction (EF), end-diastolic volume (EDV), end-systolic volume (ESV), cardiac output, and LV mass. A total of $66.66 \%(n=20)$ patients in our study group showed severely impaired EF. In addition, $26.66 \%(n=8)$ had moderately impaired $\mathrm{EF}$ while $3.3 \%(n=1)$ showed mildly impaired $\mathrm{EF}$ as evaluated by MR. Mean EF calculated by CMR in our study was $24.43 \%$, while the mean EF documented by ECG was $28.66 \%$. There was a significant positive correlation $(r=$ 0.73 ) for EF measured by ECG and CMR in all patients.

A total of $61.5 \%(n=18)$ patients in the study population showed severe dilatation involving the RV or LV. We found an association between $\mathrm{EF}$ and severity of chamber dilatation with severe impairment of $\mathrm{EF}$ being seen in all eight patients with severe biventricular enlargement.

All eight patients with moderate or severe mitral regurgitation had associated enlargement of left atrium (LA) and $\mathrm{LV}$, while of the 10 patients with mild MR, 5 patients had dilatation of LA and LV, whereas 5 patients showed only LA dilatation (-Table 1). CMR showed severely reduced EF in $90 \%$ patients who had mild or moderate mitral regurgitation. Pulmonary regurgitation with dilated main pulmonary artery and increased biventricular and right atrial diameter was found on CMR in one patient in our study group. 
Table 1 Chamber dilatation and valvular abnormality assessed by CMR

\begin{tabular}{|l|l|l|l|l|l|l|}
\hline \multirow{2}{*}{} & \multicolumn{3}{|c|}{ Chamber dilatation } & \multicolumn{2}{c|}{ Associated valvular abnormalities associated with chamber dilatation } \\
\cline { 2 - 8 } & LA & LV & $\begin{array}{l}\text { Biventricular } \\
\text { dilatation }\end{array}$ & $\begin{array}{l}\text { Mitral } \\
\text { regurgitation }\end{array}$ & $\begin{array}{l}\text { Tricuspid } \\
\text { regurgitation }\end{array}$ & Pulmonary regurgitation \\
\hline Mild & 7 & 5 & & 10 & 5 & 1 \\
\hline Moderate & 3 & 2 & & 7 & 5 & 1 \\
\hline Severe & 9 & 16 & 8 & 1 & & \\
\hline
\end{tabular}

Abbreviation: CMR, cardiac magnetic resonance; LA, left atrial; LV, left ventricle

Delayed imaging of the myocardium was performed using IR sequences 12 to 15 minutes after dynamic first-pass injection of gadolinium-based contrast in all 30 patients of DCM. LGE of the myocardium was seen in $63.33 \%(n=19)$ patients with absent myocardial enhancement in $36.66 \%(n=11)$ patients in the study group. Of the $63.33 \%$ patients who exhibited LGE, enhancement was seen in the coronary artery distribution in $10 \%$ patients enabling identification of ischemia as the cause of DCM while enhancement in a noncoronary artery distribution was seen in $53.33 \%$ patients. Thus, based on the pattern of distribution of LGE on CMR, 27 patients out of total 30 were etiologically classified as NIDCM while 3 were grouped as ischemic DCM. On clinical correlation, all three had a history of MI/angina and ECG changes.

Of the 27 patients placed in the NIDCM group, further stratification into primary NIDCM $(n=20)$ and secondary $\operatorname{NIDCM}(n=7)$ was done on the correlation of clinical history and other investigations. The primary NIDCM group composed of 20 patients and of these 4 patients with a family history of heart disease were categorized into familial etiology; 1 patient with a history of fever preceding cardiac symptoms was identified with infective viral myocarditis; while the remaining 15 patients were placed into idiopathic NIDCM as no cause was identified for the onset of the symptoms.

The secondary group of NIDCM patients $(n=7)$ included four patients with a history of metabolic disease (diabetes), three patients with a history of chronic alcoholism, and one patient of endomyocardial fibrosis (infiltrative myocardial disease) as proven on histopathological studies.

Out of the 53.33\% showing presence of LGE $(n=16)$ who were placed in the NIDCM category, diffuse LGE pattern of myocardial enhancement was seen in $40 \%(n=12), 6.6 \%$ ( $n$ $=2$ ) had mid-myocardial wall enhancement, while a combination pattern was seen in $6.6 \%$ with $3.3 \%$ patients (1) having focal enhancement of endocardium adjacent to atrioventricular (AV) valves and 3.3\% patients having focal enhancement of papillary muscles along with diffuse enhancement of myocardium on LGE.

The results of correlation of LGE with prognosis in the study group showed that $40 \%$ of patients with LGE had a worse clinical outcome of primary end point (number of hospitalizations [ $n=12]$ and sudden cardiac death $[n=2]$ ), while $43.33 \%$ patients had a higher incidence of secondary end point in the form of arrhythmias (ventricular extrasystoles and tachycardia) $(n=13)$, heart failure $(n=5)$, and increased morbidity $(n=12)$. The therapeutic management in patients with LGE detected on CMR differed from those showing focal enhancement; aggressive pharmacotherapy with digoxin in 8 patients and multidrug therapy with ACE inhibitors and angiotensin receptor blockers was instituted in 12 patients.

\section{Discussion}

The clinical presentation of DCM is characterized by progressive cardiac failure and the long-term prognosis is poor. Sudden cardiac death is known to occur due to sustained ventricular tachycardia and ventricular fibrillation. Independent of the underlying cause, a progressive increase in diameter and volume of the LV or both ventricles is the typical morphological feature in DCM, leading to impaired systolic function. The LV dysfunction in DCM is not secondary to concomitant coronary artery disease or abnormal loading conditions such as valvular heart disease or hypertension. Late gadolinium-enhanced MR helps in better therapeutic triage in DCM patients by differentiating between ischemic and nonischemic etiology. This differentiation is possible by the identification of typical patterns of myocardial enhancement of ischemic scarred myocardium and of diffuse or focal myocardial fibrosis on CMR.

DCM is characterized by an increase in ventricular chamber size independent of the underlying etiology. CMR has the ability to quantify this chamber enlargement appropriately. Chamber enlargement is the first compensatory step aiming to maintain an appropriate stroke volume (SV) as shown by the Frank-Starling mechanism. ${ }^{7}$ Above a critical sarcomere stretch, the efficiency of interaction between actin and myosin filaments decreases, causing eccentric ventricular hypertrophy, with decreased wall thickness to chamber diameter ratio and increased ventricular sphericity resulting in impairment of SV. These changes cause an increase in myocardial wall stress with increased oxygen demand and subsequent worsening of the LV systolic performance. ${ }^{10}$ Increased left atrial size is a simple "global" marker of the overall duration and severity of diastolic dysfunction. ${ }^{11}$

The normal value for both right and left atrial end-systolic diameters is less than $4 \mathrm{~cm}$. The normal LV end-diastolic diameter is less than $5.6 \mathrm{~cm} .{ }^{12}$ The RV end-diastolic diameter should be less than that of the LV end-diastolic diameter. Left atrial enlargement was seen in 19 patients in this study. We were able to diagnose LV dilatation in 23 patients (76.6\%) in our study group. In patients with severe dilatation of LV, $87.5 \%$ of patients showed NYHA class III symptomatology (-Fig. 1A, B). 


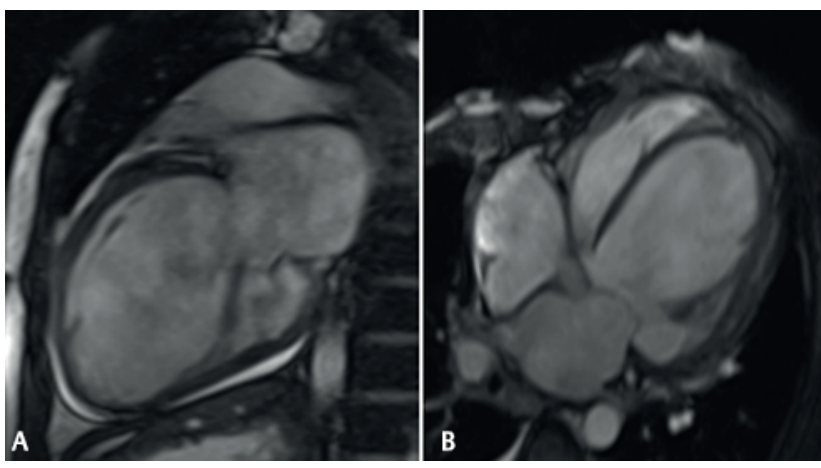

Fig. 1 (A and B): (A) Two-chamber gradient SSPE CMR image showing dilated left atrium and left ventricle along with mild pericardial effusion. (B) Marked dilatation of left ventricle and both the atria seen in this four-chamber view of SSPE CMR, in a 65-year-old male who presented with NYHA class III dyspnea of 6 months duration. The EF was found to be severely reduced on evaluation by inbuilt Argus software. CMR, cardiac magnetic resonance; EF, ejection fraction; NYHA, New York Heart Association; SSPE, subacute sclerosing panencephalitis.

Evaluation of cardiac function by CMR in DCM patients allows calculation of EDV and ESV as well as accurate estimation of EF by evaluating systolic and diastolic functions. The ventricular EDV and ESV are measured and ventricular mass is calculated using a simple acquisition of 3D stack of contiguous short-axis cine images with full biventricular coverage using semiautomated software. Increase in LV mass in DCM patients is associated with poor cardiovascular outcome and greater incidence of sudden cardiac death. A total of $60 \%$ of patients in our study group showed increased LV mass. The average LV mass calculated by CMR in DCM patients was $132.96 \mathrm{~g}$ with minimum $\mathrm{LV}$ mass recorded being $55 \mathrm{~g}$ and maximum LV mass being $202 \mathrm{~g}$.

$\mathrm{EF}$ and SV are the two important functional parameters that can be measured in DCM patients by CMR with the help of the following equations:

$$
\begin{gathered}
\mathrm{SV}=\mathrm{EDV} \mathrm{ESV} \\
\mathrm{EF}=\mathrm{SV} / \mathrm{EDV}
\end{gathered}
$$

Out of the 30 patients of DCM in our study group evaluated by CMR, $43.3 \%$ patients $(n=13)$ showed severely impaired EDV while $70 \%$ patients showed severely impaired ESV. Mean EDV measured by CMR in the study population was $167.3 \mathrm{~mL}$ and mean ESV measured was $130.11 \mathrm{~mL}$ (-Fig. 2A, B).

Myocardial contractile dysfunction with global hypokinesia or regional wall motion abnormalities are often seen in patients of DCM due to significant strain on the myocardium. Ventricular dyssynchrony is diagnosed on CMR when the diminished systolic wall thickness is seen along with wall motion abnormalities in the form of hypokinesia, dyskinesia, or akinesia ( - Fig. 3). Myocardial thinning is a useful indicator of LV dysfunction. LV dysfunction is reliably diagnosed on CMR if the wall thickness to chamber radius ratio is found to be reduced. ${ }^{13}$ Black blood images allow assessment of ventricular wall thickness.

In this study, wall motion abnormalities were diagnosed by CMR in $70 \%(n=21)$ patients of suspected DCM. We found an association between the severity of LV dysfunction and presence of wall motion abnormalities. A total of $81 \%$ of patients $(n=17)$ who showed global/regional wall motion abnormalities (WMA) on CMR had severely impaired $\mathrm{EF}(<35 \%)$ in our study (- Table 2 ).

We found a positive correlation between the presence of wall motion abnormalities and poor EF (Chi-square: $11.42, p$ $=0.000723$ ).

Fibrosis in DCM is a result of myocardial inflammation as well as microvascular ischemia. ${ }^{14,15} \mathrm{~A}$ high proportion of patients of DCM show evidence of myocardial fibrosis with myocyte apoptosis and hypertrophy of the remaining myocytes occurs resulting in chamber dilatation and marked impairment of LV function. ${ }^{16}$ Additionally, there is excessive collagen deposition and decreased capillary density in the myocardium which produces both reactive (interstitial and
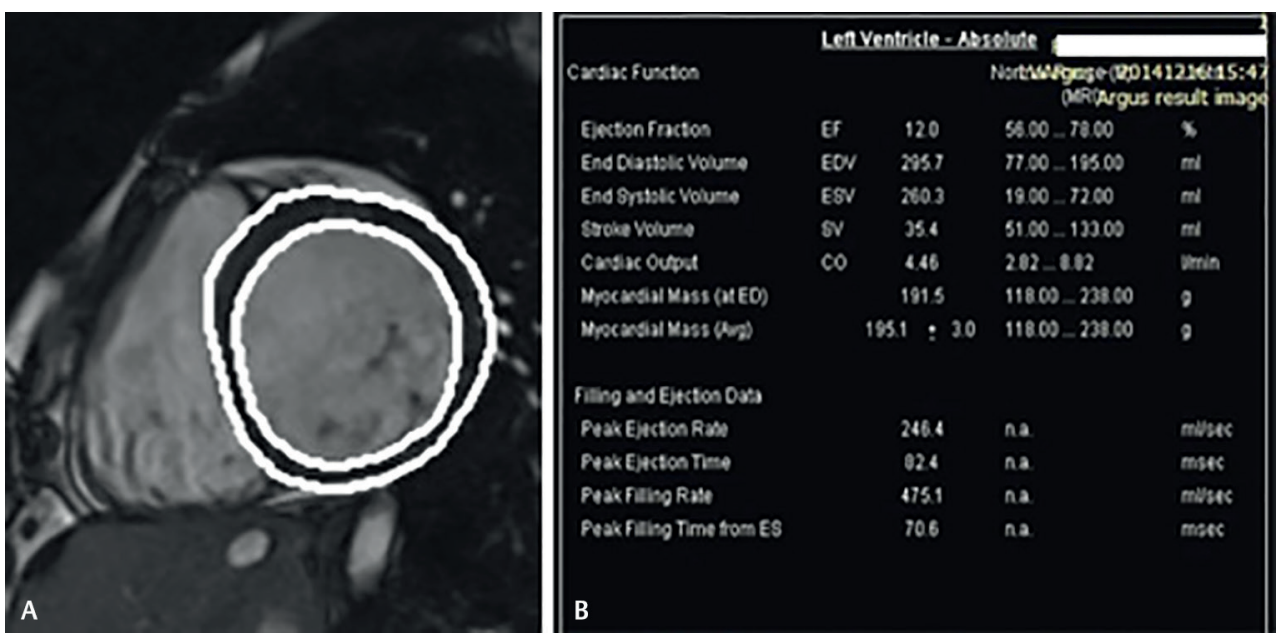

Fig. 2 (A and B) Evaluation of LV function by drawing epicardial and endocardial contours on left ventricle short axis. Cine end-diastolic CMR image shows the stroke volume to be extremely low $(35.4 \mathrm{~mL})$ in this patient of DCM who presented with nonsustained ventricular tachycardia on ECG. CMR detected severe LV dilatation, and various volume indices calculated by Argus software are shown, with a severely reduced ejection fraction of $12 \%$, increased end-systolic volume of $260.3 \mathrm{~mL}$, and raised end-diastolic volume of $295.7 \mathrm{~mL}$. CMR, cardiac magnetic resonance; DCM, dilated cardiomyopathy; LV, left ventricle. 


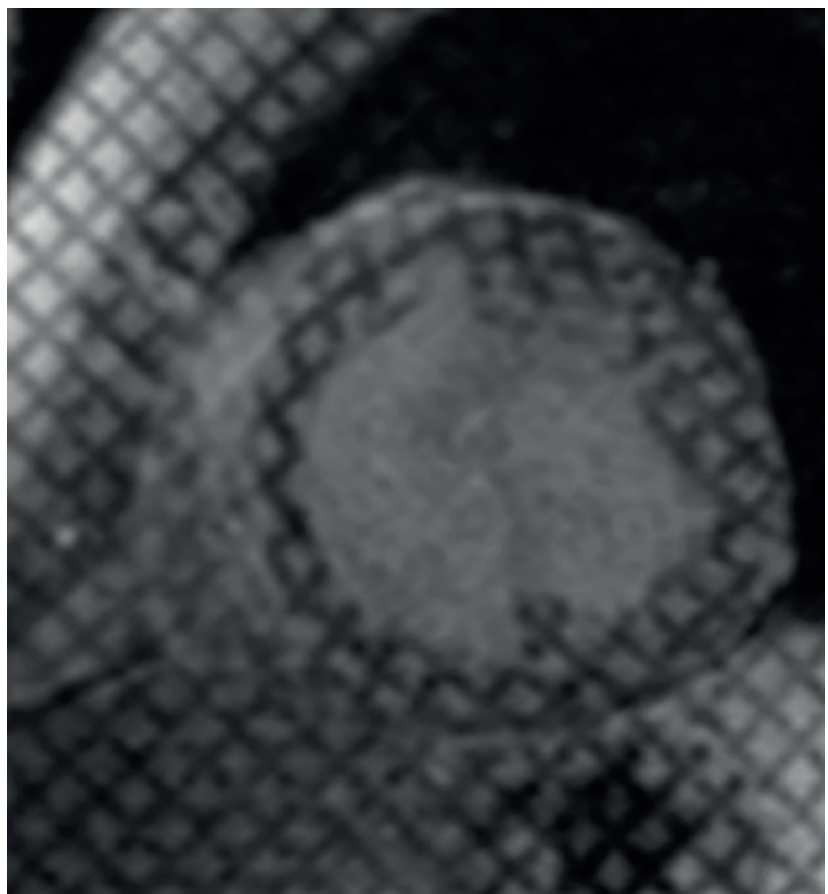

Fig. 3 Myocardial grid tagged CMR image shows regional wall motion abnormality with LV dyssynchrony in the anteroseptal region in a patient of idiopathic DCM with NYHA II symptoms. CMR, cardiac magnetic resonance; DCM, dilated cardiomyopathy; LV, left ventricle; NYHA, New York Heart Association.

Table 2 CMR assessment of wall motion abnormalities

\begin{tabular}{|l|l|l|}
\hline $\begin{array}{l}\text { Wall motion } \\
\text { abnormalities }\end{array}$ & \multicolumn{2}{|c|}{ Number of patients } \\
\hline Global hypokinesia & 9 & \\
\hline Regional wall motion & 12 & $\begin{array}{l}\text { Anteroseptal and anterolateral } \\
\text { wall (9) }\end{array}$ \\
\hline Abnormalities & & Inferior wall (3) \\
\hline No hypokinesia & 9 & \\
\hline
\end{tabular}

Abbreviation: CMR, cardiac magnetic resonance.

perivascular) and reparative (replacement) fibrosis. This fibrosis in turn causes increased wall stress with global hypokinesia. These changes are accompanied by progressive LV remodeling thereby increasing the metabolic demands and augmenting the microvascular ischemia as well as focal myocyte necrosis.

Myocardial fibrosis can be delineated on LGE CMR in form of focal or diffuse delayed myocardium enhancement because of abnormal washout kinetics of gadolinium. The normal myocardium does not show delayed enhancement on CECMR while fibrosed myocardium retains the gadolinium contrast on for 10 to 12 minutes. The presence of LGE shown by CMR in DCM is an indicator of myocardial fibrosis and the typical pattern is of a characteristic linear stripe of enhancement in the mid-wall ( - Fig. 4 ) or a diffuse transmural enhancement (-Fig. 5). This mid-wall fibrosis has been described due to a combination of factors including genetic predisposition, exposure to toxins or pathogens (viral, bacterial), as well as abnormal modulation of immune and metabolic responses such as overactivity of the renin angiotensin

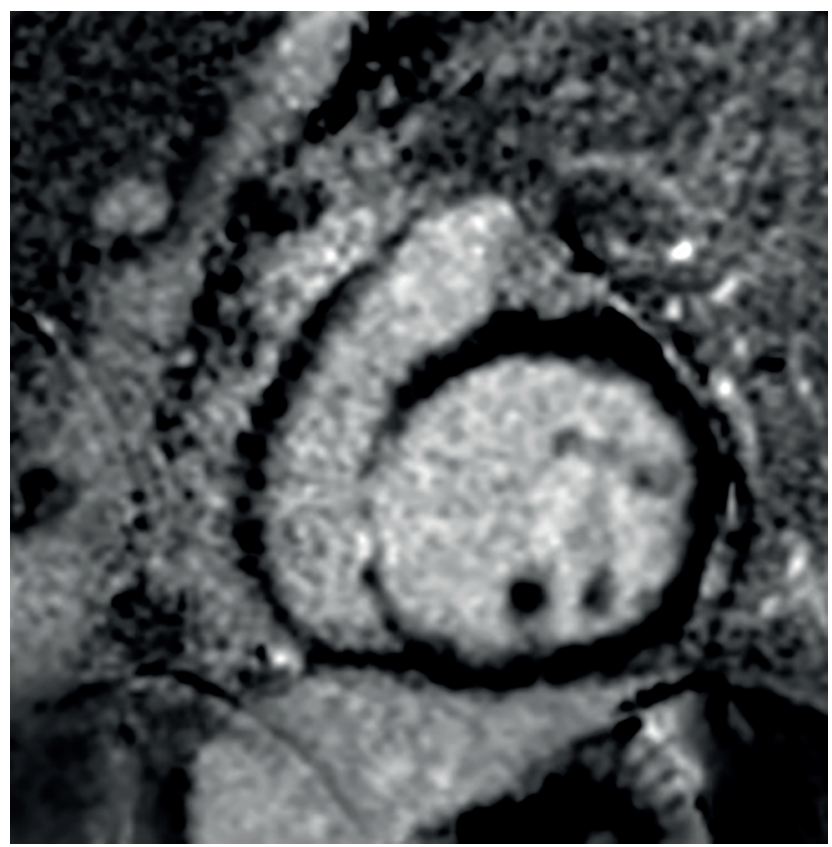

Fig. 4 Delayed post-gadolinium IR-prepared acquisition at 12 minutes in the four-chamber plane shows characteristic mid-wall stripe of enhancement of the LV myocardium along with significant LV dilatation, in a patient of idiopathic DCM. DCM, dilated cardiomyopathy; LV, left ventricle.

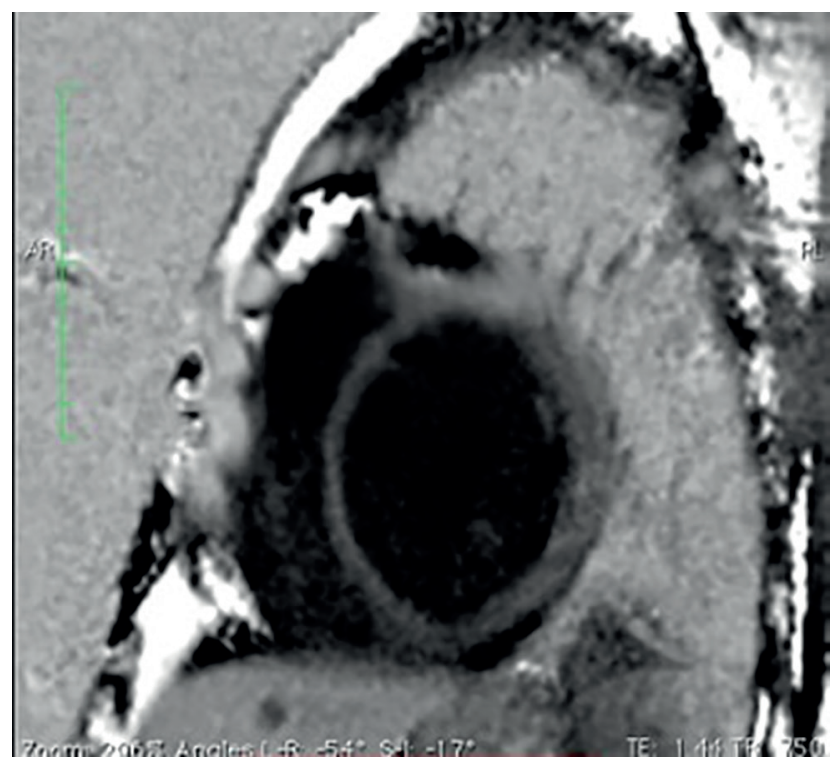

Fig. 5 LV myocardium is seen diffusely enhancing in this four-chamber delayed CMR image acquired at 15 minutes post-gadolinium injection in a patient of muscular dystrophy with primary DCM. CMR, cardiac magnetic resonance; DCM, dilated cardiomyopathy.

aldosterone system. In our study diffuse transmural pattern of enhancement was seen in 12 patients while a mid-wall pattern of enhancement was found in two patients (6.6\%). A combination pattern of enhancement in the form of diffuse transmural enhancement along with focal enhancement adjacent to $A V$ valves and papillary muscle enhancement was seen in two patients. LGE in coronary artery territory was seen in 3 patients (10\%) out of 30, indicating ischemic etiology of DCM in these patients. 
In this study, we found a correlation between impairment of ventricular function and presence of LGE of myocardium, a definite indicator of fibrosis. Of the 19 patients who showed LGE, 17 patients also had severely impaired $\mathrm{EF}(<35 \%)$ as detected by CMR while 1 patient had mildly impaired EF. The results of this study show that the more definitive the presence of fibrosis, as evidenced by typical patterns of LGE, more severe is the extent of LV dysfunction.

The first aim in DCM patients is to exclude coronary artery disease as the underlying cause of myocardial damage and dysfunction, ${ }^{8,17,18}$ Differentiation between the ischemic and nonischemic causes of DCM has important therapeutic implications with management in each subgroup being different. Patients with ischemic DCM benefit from modification of risk factors, drug therapy with $\beta$-blockers, myocardial stenting, or coronary artery bypass surgery, whereas patients with NIDCM are better candidates for implantation cardiac devices or even for transplantation. As discussed earlier the patterns of LGE on CMR enable this crucial differentiation of ischemic from nonischemic causes of DCM.

Enhancement patterns further differentiate amongst the nonischemic etiologies of DCM. Viral myocarditis is the etiological factor for NIDCM in $10 \%$ patients and histopathological studies of patients with viral myocarditis have confirmed the presence of inflammatory exudates in the myocardium, as well as fibrosis. ${ }^{19}$ LGE enhancement of myocardium is seen typically in the epicardial location in these patients (-Fig. 6A). Another causative factor of DCM is endomyocardial fibrosis and on LGE scans diffuse enhancement of the myocardium along with focal enhancement is seen typically adjacent to the AV valve in patients of endomyocardial fibrosis (EMF) ( - Fig. 6B). Idiopathic NIDCM group is identified when, along with the mid-wall or diffuse transmural enhancement, marked LV dilatation and diffuse thinning of myocardium are also present. In our study, we found idiopathic NIDCM in 15 patients (50\%). The diffuse pattern of LGE is more often seen in the idiopathic subgroup of NIDCM patients and also in the primary familial subgroup.

Thus, in NIDCM, the pattern and location of LGE is distinctively different and does not correspond to a specific coronary artery territory.

In the ischemic group of DCM, the most vulnerable zone for ischemia is the subendocardium. Enhancement of the myocardium in the subendocardial layer is seen on LGE images corresponding to specific coronary artery territory which may be LAD, LCX or RCA, or an overlap of these territories ( - Fig. 7 ).

Phase-contrast cine MRI is an accurate technique for quantifying the severity of valvular regurgitation associated with LV dilatation and providing information on diastolic function. The LV enlargement further dilates the mitral valve ring, dislocates the papillary muscles, and impairs leaflet coaptation thereby causing mitral valvular regurgitation and putting additional volume load on the already dilated ventricle. ${ }^{7}$ This in turn will lead to further lowering of EF by compounding the failure.

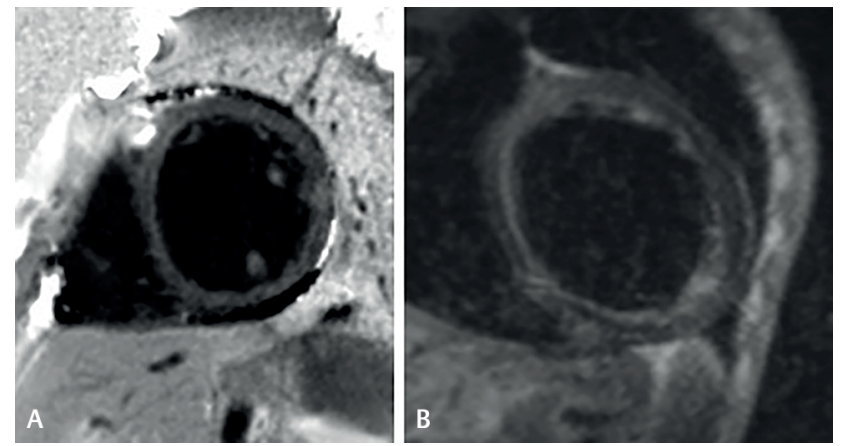

Fig. 6 (A and B): (A) Diffuse enhancement of myocardium along with enhancement of papillary muscles in an 18-year-old male presenting with fever and acute-onset dyspnea. The histopathological diagnosis after endomyocardial biopsy was of myocarditis due to viral etiology. This LGE pattern of papillary muscle enhancement is typical of myocarditis. (B) IR T1 image showing diffuse LGE of the myocardium mainly in the subendocardial region is seen in endomyocardial fibrosis in a 32-year-old male with NYHA II symptoms. LGE, late gadolinium enhancement; NYHA, New York Heart Association.

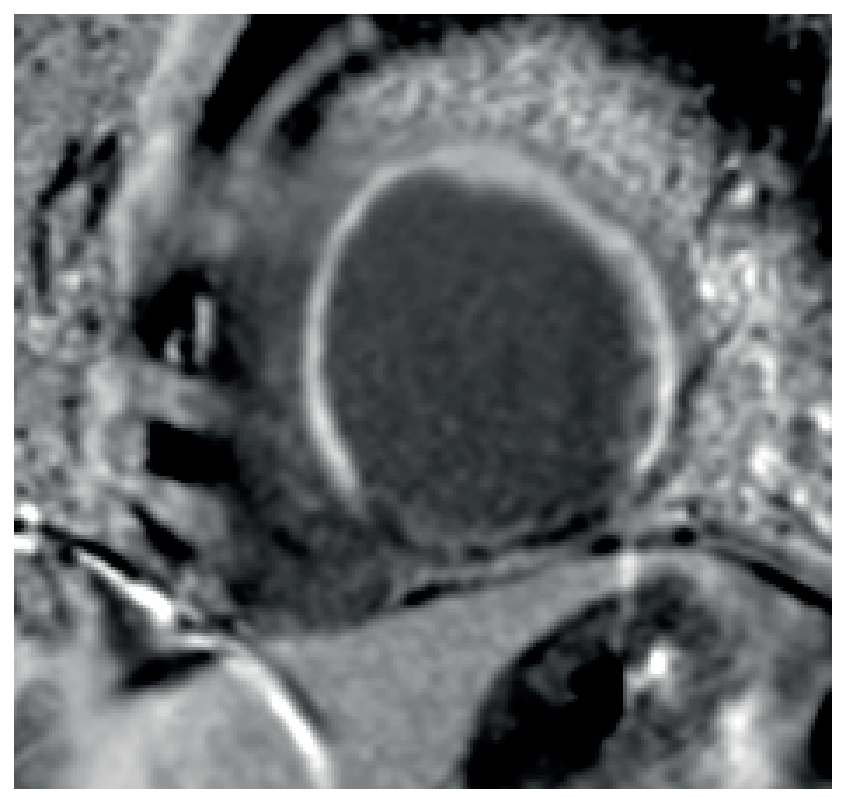

Fig. 7 Delayed enhancement of myocardium typically in LAD territory is depicted in a patient with clinical presentation of dyspnea and history of angina 2 years back. A diagnosis of ischemic etiology of DCM was established on the basis of pattern of LGE on CMR. DCM, dilated cardiomyopathy; LAD, left anterior descending; LGE, late gadolinium enhancement.

In this study, in all patients of biventricular dilatation (RV and LV) severe mitral regurgitation was present and 60\% of patients of moderate mitral regurgitation also showed biventricular dilatation. An associated tricuspid regurgitation of the same severity was also detected in $80 \%$ of the patients in whom moderate to severe mitral regurgitation was present and $30 \%$ of the patients having mild mitral regurgitation showed an associated mild tricuspid regurgitation.

Intracardiac thrombi can be present as a complication of DCM. These are diagnosed as a soft-tissue well-marginated intracavitary mass of variable signal intensity. They do not 


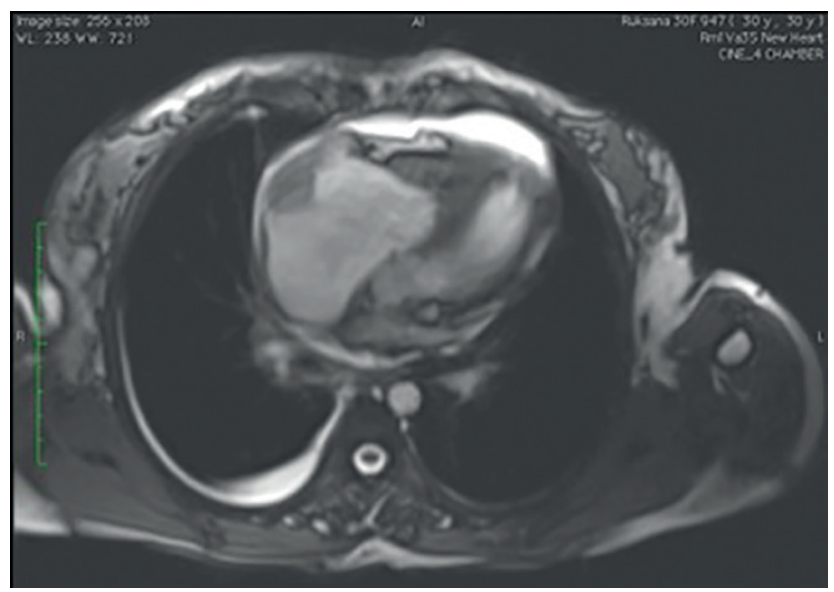

Fig. 8 Mural right atrial thrombus seen as a hypointense nonenhancing lesion, a complication of DCM detected by CMR along with pericardial and pleural effusion. CMR, cardiac magnetic resonance; DCM, dilated cardiomyopathy.

show enhancement on first pass or delayed CECMR. CMR has an excellent sensitivity and specificity for thrombus detection and is superior to ECG for characterizing intracardiac masses. In this study group of DCM patients intracardiac thrombus was present in $3 \%$ patients.

Pericardial or pleural effusion is sometimes associated with DCM and these can be well visualized on CMR. Pleural effusion as well as pericardial effusion was seen in one patient (3\%) in this study group ( - Fig. 8).

\section{Conclusion}

CMR is considered the most important imaging modality for diagnosis of DCM as it allows precise and reliable assessment of morphology and characterization of myocardial fibrosis, with simultaneous evaluation of extent of ventricular dysfunction and dyssynchrony. Quantification of ventricular dysfunction assists in adequate therapeutic triage and prediction of prognosis. CMR enables evaluation of cardiac function in patients of DCM by calculating EDV, ESV and EF, and reliably grades dysfunction into mild, moderate, and severe categories, which have prognostic significance. The cardiac function and EF calculated by CMR are more reliable than that evaluated by any other modality. CMR allows greater accuracy in reliable differentiation of ischemic and nonischemic etiologies of DCM based on the pattern of LGE, and is extremely useful in identification of certain etiologies such as myocarditis and endomyocardial fibrosis. CMR findings greatly assist in risk stratification, prognosis, and planning of appropriate therapy in DCM patients.

\section{Financial Support and Sponsorship}

Nil.

\section{Conflicts of Interest}

There are no conflicts of interest.

\section{References}

1 Maron BJ, Towbin JA, Thiene G, et al. American Heart AssociationCouncil on Clinical Cardiology, Heart Failure and Transplantation CommitteeQuality of Care and Outcomes Research and Functional Genomics and Translational Biology Interdisciplinary Working GroupsCouncil on Epidemiology and Prevention. Contemporary definitions and classification of the cardiomyopathies: an American Heart Association Scientific Statement from the Council on Clinical Cardiology, Heart Failure and Transplantation Committee; Quality of Care and Outcomes Research and Functional Genomics and Translational Biology Interdisciplinary Working Groups; and Council on Epidemiology and Prevention. Circulation 2006;113(14):1807-1816

2 Mestroni L, Brun F, Spezzacatene A, Sinagra G, Taylor MR. Genetic causes of dilated cardiomyopathy. Prog Pediatr Cardiol 2014;37(1-2):13-18

3 Elliott P, Andersson B, Arbustini E, et al. Classification of the cardiomyopathies: a position statement from the European Society Of Cardiology Working Group on Myocardial and Pericardial Diseases. Eur Heart J 2008;29(2):270-276

4 Jefferies JL, Towbin JA. Dilated cardiomyopathy. Lancet 2010; 375(9716): :752-762

5 Charron P, Arad M, Arbustini E, et al; European Society of Cardiology Working Group on Myocardial and Pericardial Diseases. Genetic counselling and testing in cardiomyopathies: a position statement of the European Society of Cardiology Working Group on Myocardial and Pericardial Diseases. Eur Heart J 2010;31(22):2715-2726

6 Dec GW, Fuster V. Idiopathic dilated cardiomyopathy. N Engl J Med 1994;331(23):1564-1575

7 Slavich M, Florian A, Bogaert J. The emerging role of magnetic resonance imaging and multidetector computed tomography in the diagnosis of dilated cardiomyopathy. Insights Imaging 2011;2(4):453-469

8 McCrohon JA, Moon JC, Prasad SK, et al. Differentiation of heart failure related to dilated cardiomyopathy and coronary artery disease using gadolinium-enhanced cardiovascular magnetic resonance. Circulation 2003;108(1):54-59

9 Belloni E, De Cobelli F, Esposito A, et al. MRI of cardiomyopathy. AJR Am J Roentgenol 2008;191(6):1702-1710

10 Fujita N, Duerinekx AJ, Higgins CB. Variation in left ventricular regional wall stress with cine magnetic resonance imaging: normal subjects versus dilated cardiomyopathy. Am Heart J 1993;125(5, Pt 1) :1337-1345

11 Pritchett AM, Mahoney DW, Jacobsen SJ, Rodeheffer RJ, Karon BL, Redfield MM. Diastolic dysfunction and left atrial volume: a population-based study. J Am Coll Cardiol 2005;45(1):87-92

12 Lang RM, Bierig M, Devereux RB, et al; Chamber Quantification Writing Group; American Society of Echocardiography's Guidelines and Standards Committee; European Association of Echocardiography. Recommendations for chamber quantification: a report from the American Society of Echocardiography's Guidelines and Standards Committee and the Chamber Quantification Writing Group, developed in conjunction with the European Association of Echocardiography, a branch of the European Society of Cardiology. J Am Soc Echocardiogr 2005;18(12):1440-1463

13 Barison A, Masci PG, Emdin M. Fibrosis and mortality in patients with dilated cardiomyopathy. JAMA 2013;309(24):2547

14 Mann DL, Bristow MR. Mechanisms and models in heart failure: the biomechanical model and beyond. Circulation 2005;111(21):2837-2849 
15 Baig MK, Mahon N, McKenna WJ, et al. The pathophysiology of advanced heart failure. Am Heart J 1998;135(6, Pt 2 Suppl):S216-S230

16 Beltrami CA, Finato $\mathrm{N}$, Rocco $\mathrm{M}$, et al. The cellular basis of dilated cardiomyopathy in humans. J Mol Cell Cardiol 1995;27(1):291-305

17 Wu E, Judd RM, Vargas JD, Klocke FJ, Bonow RO, Kim RJ. Visualisation of presence, location, and transmural extent of healed Q-wave and non-Q-wave myocardial infarction. Lancet 2001;357(9249):21-28
18 Koito H, Suzuki J, Ohkubo N, Ishiguro Y, Iwasaka T, Inada M. Gadolinium-diethylenetriamine pentaacetic acid enhanced magnetic resonance imaging of dilated cardiomyopathy: clinical significance of abnormally high signal intensity of left ventricular myocardium [in Japanese]. J Cardiol 1996;28(1):41-49

19 de Leeuw N, Ruiter DJ, Balk AH, de Jonge N, Melchers WJ, Galama JM. Histopathologic findings in explanted heart tissue from patients with end-stage idiopathic dilated cardiomyopathy. Transpl Int 2001;14(5):299-306 